\title{
Automatic Identification of the Number and Values of Decision Thresholds in the Log-Ratio Image for Change Detection in SAR Images
}

\author{
Yakoub Bazi, Student Member, IEEE, Lorenzo Bruzzone, Senior Member, IEEE, and Farid Melgani, Member, IEEE
}

\begin{abstract}
In this letter, we propose an extension of an automatic and unsupervised change-detection method for synthetic aperture radar images we presented earlier. By analyzing a properly defined cost function, the proposed method allows the automatic detection of the number (zero, one, or two) and the values of the decision thresholds associated with changes (if any) in the log-ratio image. This cost function is the minimum value of the criterion function adopted to select the decision threshold in the log-ratio image according to a modified double-thresholding Kittler-Illingworth algorithm (implemented under the generalized Gaussian assumption for changed and unchanged classes). Experimental results carried out both on real and simulated multitemporal synthetic aperture radar images proved the effectiveness of the proposed automatic method in detecting both the number of changes to be identified (the situation of no changes is also explicitly identified) and the related threshold values.
\end{abstract}

Index Terms-Change detection, double thresholding, synthetic aperture radar (SAR) images.

\section{INTRODUCTION}

I $\mathrm{N}$ earlier work [1], we presented an automatic and unsupervised change-detection method suitable for the analysis of single-channel single-polarization multitemporal synthetic aperture radar (SAR) images. This method is based on three main sequential steps: 1) controlled preprocessing based on adaptive filtering (despeckling); 2) comparison of multitemporal images according to a log-ratio operator; and 3) automatic thresholding of the log-ratio image. The first step aims at reducing speckle noise in a controlled way in order to maximize the separability between changed and unchanged classes. In the second step, the two filtered images are compared in order to generate the log-ratio image. Finally, in the third step, changes are identified by analyzing the log-ratio image according to a novel thresholding technique. The latter is based on the Kittler-Illingworth (KI) threshold selection method [2] (originally developed in the Gaussian hypothesis), reformulated under the generalized Gaussian (GG) assumption for the changed and unchanged classes.

However, in the above-mentioned analysis (as usually done in the context of change detection) changes are assumed to be on one side of the histogram of the log-ratio image, which is not true for all change-detection problems. In particular, changes may be present on both sides of the histogram of the

Manuscript received April 29, 2005; revised November 2, 2005. This research was supported by the Italian Ministry of Education, University, and Research.

The authors arewith the Department of Information and Communication Technologies, University of Trento, I-38050 Trento, Italy (email: lorenzo.bruzzone@ing.unitn.it; melgani@dit.unitn.it).

Digital Object Identifier 10.1109/LGRS.2006.869973

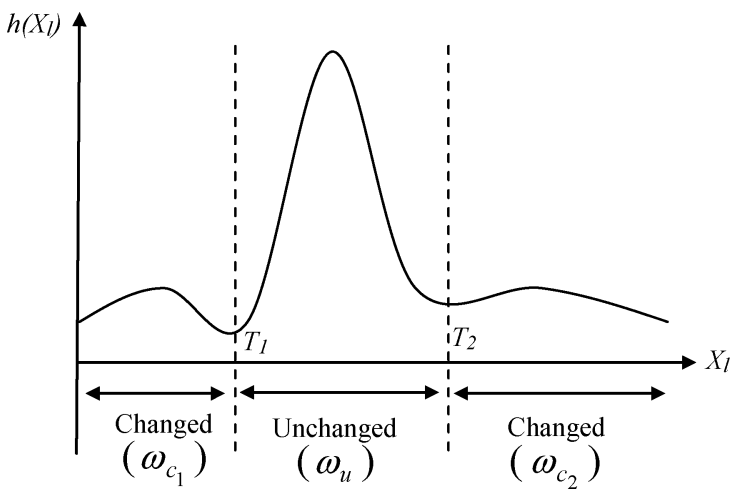

Fig. 1. Histogram of the log-ratio image characterized by a double change on both sides of the unchanged class.

log-ratio image. In order to address this critical issue, in this letter we propose to determine automatically the number and values of decision thresholds (one or two) corresponding to one or more typologies of changes by analyzing a simple cost function. The latter is the minimum value of the criterion function obtained by thresholding the log-ratio image with the modified double-thresholding KI algorithm under the GG assumption for changed and unchanged classes. The proposed technique can also automatically detect situations in which no changes occurred between the considered multitemporal SAR images.

The letter is organized into four sections. The problem formulation and the automatic analysis of the log-ratio image according to the modified double-thresholding KI algorithm is presented in Section II. The experimental results are reported in Section III. Finally, conclusions are drawn in Section IV.

\section{Problem Formulation AND Description OF THE PROPOSED METHOD}

Let us consider two coregistered SAR intensity (or amplitude) images $X_{1}=\left\{X_{1}(i, j), 1 \leq i \leq I, 1 \leq j \leq J\right\}$ and $X_{2}=\left\{X_{2}(i, j), 1 \leq i \leq \bar{I}, 1 \leq j \leq \bar{J}\right\}$ acquired over the same geographical area at two different times $t_{1}$ and $t_{2}$, respectively. Let $X_{l}$ be the log-ratio image obtained by applying the log-ratio operator to $X_{1}$ and $X_{2}$ (i.e., $X_{l}(i, j)=$ $\left.\log \left[X_{1}(i, j) / X_{2}(i, j)\right]\right)$, and let $h\left(X_{l}\right)$ be its corresponding histogram, where $X_{l} \in[0, L-1]$ ( $L$ stands for the number of possible gray levels).

First, we analyze the cost function defined to discriminate between changed and unchanged classes in the histogram of the log-ratio image (see Fig. 1). In this context, the histogram $h\left(X_{l}\right)$ is viewed as a mixture of three distributions (two distributions 
are associated with the changed class $\omega_{c}=\omega_{c_{1}} \cup \omega_{c_{2}}$ and one with the unchanged class $\omega_{u}$ ), i.e.,

$$
h\left(X_{l}\right)=P\left(\omega_{u}\right) p\left(X_{l} \mid \omega_{u}\right)+\sum_{i=1}^{2} P\left(\omega_{c_{i}}\right) p\left(X_{l} \mid r \omega_{c_{i}}\right)
$$

where $P\left(\omega_{c_{i}}\right)$ and $P\left(\omega_{u}\right)$ are the prior probabilities and $p\left(X_{l} \mid \omega_{c_{i}}\right)$ and $p\left(X_{l} \mid \omega_{u}\right)$ are the class-conditional densities associated with $\omega_{c_{i}}(i=1,2)$ and $\omega_{u}$, respectively. The latter are assumed to follow a GG distribution, i.e.,

$$
p\left(X_{l} \mid \omega_{i}\right)=a_{i} e^{-\left[b_{i}\left|X_{l}-m_{i}\right|\right]^{\beta_{i}}}, \quad i=\left(u, c_{1}, c_{2}\right)
$$

where the positive constants $a_{i}$ and $b_{i}$ are given by

$$
a_{i}=\frac{b_{i} \beta_{i}}{2 \Gamma\left(\frac{1}{\beta_{i}}\right)} \quad b_{i}=\frac{1}{\sigma_{i}} \sqrt{\frac{\Gamma\left(\frac{3}{\beta_{i}}\right)}{\Gamma\left(\frac{1}{\beta_{i}}\right)}} .
$$

The terms $m_{i}, \sigma_{i}^{2}$, and $\beta_{i}$ are the mean, the variance, and the shape parameters of the distribution, respectively, and $\Gamma($.$) is$ the Gamma function.

Second, we address the problem of determining the number of changes present in the scene (i.e., the number of decision thresholds) by exploiting the cost function yielded in the first step. The description of both steps is presented in Sections II-A and $\mathrm{B}$.

\section{A. Double-Thresholding KI Algorithm Under the GG Assumption}

This algorithm is based on the selection of an appropriate pair of threshold values $\left(T_{1}, T_{2}\right)$ that optimize a predefined criterion function $J\left(T_{1}, T_{2}\right)$, which averages a cost function $c\left(X_{l}, T_{1}, T_{2}\right)$ over the histogram $h\left(X_{l}\right)$. The cost function $c\left(X_{l}, T_{1}, T_{2}\right)$ measures the cost of classifying pixels by comparing their gray-levels $X_{l}$ with the two thresholds. The KI criterion function $J\left(T_{1}, T_{2}\right)$ is given by [2]

where

$$
J\left(T_{1}, T_{2}\right)=\sum_{X_{l}=0}^{L-1} h\left(X_{l}\right) c\left(X_{l}, T_{1}, T_{2}\right)
$$

$$
c\left(X_{l}, T\right)= \begin{cases}-2 \ln P^{c_{1}}\left(X_{l}, T_{1}, T_{2}\right), & \text { if } X_{l} \leq T_{1} \\ -2 \ln P^{u}\left(X_{l}, T_{1}, T_{2}\right), & \text { if } T_{1}<X_{l} \leq T_{2} \\ -2 \ln P^{c_{2}}\left(X_{l}, T_{1}, T_{2}\right), & \text { if } X_{l}>T_{2}\end{cases}
$$

in which $P^{i}\left(X_{l}, T_{1}, T_{2}\right)\left(i=u, c_{1}, c_{2}\right)$ are the posterior probabilities of the classes $\omega_{u}, \omega_{c_{1}}$, and $\omega_{c_{2}}$, given the gray-level $X_{l}$ and specific values of the pair of thresholds $\left(T_{1}, T_{2}\right)$. The optimal threshold values are those that minimize the following criterion function:

$$
\left(T_{1}^{*}, T_{2}^{*}\right)=\underset{\left(T_{1}, T_{2}\right)=[0,1, \ldots L-1]^{2}}{\arg \min } J\left(T_{1}, T_{2}\right) .
$$

Using the Bayes theorem, the posterior probability can be expressed in terms of the prior probability and the class-condi tional pdf, i.e.,

$$
P^{i}\left(X_{l}, T_{1}, T_{2}\right)=\frac{P\left(\omega_{i}\right) \cdot p\left(X_{l} \mid \omega_{i}, T_{1}, T_{2}\right)}{\sum_{j \in\left\{u, c_{1}, c_{2}\right\}} P\left(\omega_{j}\right) \cdot p\left(X_{l} \mid \omega_{j}, T_{1}, T_{2}\right)} .
$$

Under the GG assumption for the class conditional densities related to unchanged and changed classes, it can been shown that (4) becomes

$$
\begin{aligned}
J\left(T_{1}, T_{2}\right)= & \sum_{X_{l}=0}^{T_{1}} h\left(X_{l}\right) \cdot\left[b_{c_{1}}\left(T_{1}\right)\left|X_{l}-m_{c_{1}}\left(T_{1}\right)\right|\right]^{\beta_{c_{1}}\left(T_{1}\right)} \\
& +\sum_{X_{l}=T_{1}+1}^{T_{2}} h\left(X_{l}\right) \\
& \cdot\left[b_{u}\left(T_{1}, T_{2}\right)\left|X_{l}-m_{u}\left(T_{1}, T_{2}\right)\right|\right]^{\beta_{u}\left(T_{1}, T_{2}\right)} \\
& +\sum_{X_{l}=T_{2}+1}^{L-1} h\left(X_{l}\right) \cdot\left[b_{c_{2}}\left(T_{2}\right)\left|X_{l}-m_{c_{2}}\left(T_{2}\right)\right|\right]^{\beta_{c_{2}}\left(T_{2}\right)} \\
& +H\left(T_{1}, T_{2}\right)
\end{aligned}
$$

where

$$
\begin{aligned}
H\left(T_{1}, T_{2}\right)= & -P_{c_{1}}\left(T_{1}\right) \cdot\left[\ln P_{c_{1}}\left(T_{1}\right)+\ln a_{c_{1}}\left(T_{1}\right)\right] \\
& -P_{u}\left(T_{1}, T_{2}\right) \cdot\left[\ln P_{u}\left(T_{1}, T_{2}\right)+\ln a_{u}\left(T_{1}, T_{2}\right)\right] \\
& -P_{c_{2}}\left(T_{2}\right) \cdot\left[\ln P_{c_{2}}\left(T_{2}\right)+\ln a_{c_{2}}\left(T_{2}\right)\right] .
\end{aligned}
$$

The parameters to be estimated (which depend on the pair of threshold values $\left.\left(T_{1}, T_{2}\right) \in[0, L-1]^{2}\right)$ are the prior probabilities $P_{u}\left(T_{1}, T_{2}\right), P_{c_{1}}\left(T_{1}\right)$, and $P_{c_{2}}\left(T_{2}\right)$, the mean values $m_{u}\left(T_{1}, T_{2}\right), m_{c_{1}}\left(T_{1}\right)$, and $m_{c_{2}}\left(T_{2}\right)$, the variances $\sigma_{u}^{2}\left(T_{1}, T_{2}\right)$, $\sigma_{c_{1}}^{2}\left(T_{1}\right)$, and $\sigma_{c_{2}}^{2}\left(T_{2}\right)$, and the shapes $\beta_{u}\left(T_{1}, T_{2}\right), \beta_{c_{1}}\left(T_{1}\right)$, and $\beta_{c_{2}}\left(T_{2}\right)$ associated with the unchanged class $\omega_{u}$ and the changed classes $\omega_{c_{1}}$ and $\omega_{c_{2}}$, respectively. The above parameters are determined according to the estimation method described in [1].

\section{B. Identification of the Number of Thresholds}

The determination of the number of thresholds to be applied to the log-ratio image is a critical issue since the number of changes that occurred between the two acquisition dates is unknown a priori (we may have changes on one or two sides of the histogram but we may also have no changes at all). A possible solution to this problem is to exploit the information yielded by applying the modified double-thresholding KI algorithm to the log-ratio image. In particular, once the optimal thresholds are obtained, the next step is to decide on keeping both, rejecting one or rejecting both (no changes occurred between the two images). This is made simply by analyzing the nature of the point $\left(T_{1}^{*}, T_{2}^{*}\right)$ with respect to the function $J\left(T_{1}, T_{2}\right)$. Accordingly, three decisions can be taken: 1) the two thresholds are confirmed if $\left(T_{1}^{*}, T_{2}^{*}\right)$ is a minimum in the two-dimensional (2-D) threshold space [see Fig. 2(a)], which means that at least two typologies of changes are present in the log-ratio image; 2 ) one of the two thresholds is rejected if $\left(T_{1}^{*}, T_{2}^{*}\right)$ is not a minimum in the 2-D threshold space but the remaining one is a minimum in one-dimensional (1-D) threshold space [see Fig. 2(b)], which indicates the presence of one typology of change; and 3 ) both thresholds are rejected if $\left(T_{1}^{*}, T_{2}^{*}\right)$ is a saddle point in both 2-D and 1-D threshold spaces [see Fig. 2(c)], which means 


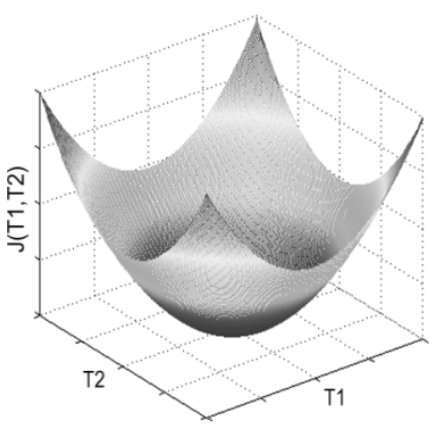

(a)

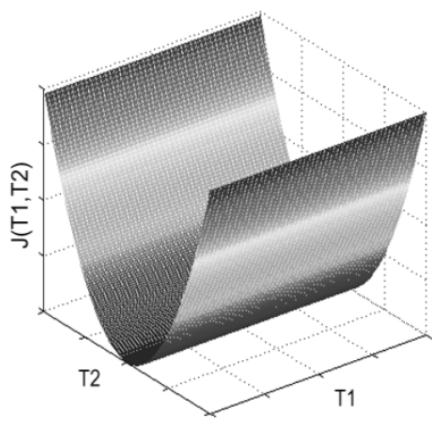

(b)

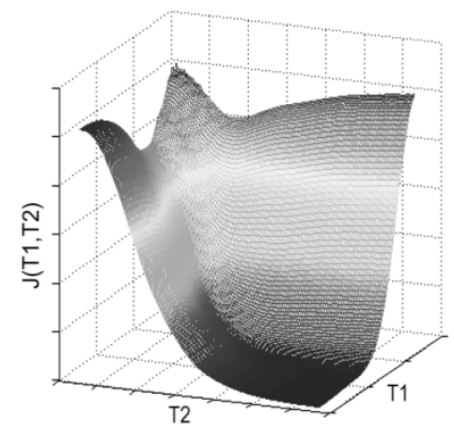

(c)

Fig. 2. Examples of the possible behaviors of $J\left(T_{1}, T_{2}\right)$ when characterized by (a) a minimum point in the 2-D threshold space, (b) a minimum point in a 1-D threshold space, and (c) a saddle point.

that no changes are present in the log-ratio image. Mathematically speaking, this problem can be solved by computing the Hessian matrix $H_{\left(T_{1}^{*}, T_{*}^{*}\right)}$ (matrix of second partial derivatives) of $J\left(T_{1}, T_{2}\right)$ at $\left(T_{1}^{*}, T_{2}^{*}\right)$, i.e.,

$$
H_{\left(T_{1}^{*}, T_{2}^{*}\right)}=\left[\begin{array}{cc}
\frac{\partial^{2} J}{\partial T_{1}^{2}} & \frac{\partial^{2} J}{\partial T_{1} \partial T_{2}} \\
\frac{\partial^{2} J}{\partial T_{2} \partial T_{1}} & \frac{\partial^{2} J}{\partial T_{2}^{2}}
\end{array}\right] .
$$

If the Hessian matrix is positive definite (i.e., $\left(T_{1}^{*}, T_{2}^{*}\right)$ is a minimum) then the two thresholds are taken into consideration. Otherwise, if $H_{\left(T_{1}^{*}, T_{2}^{*}\right)}$ is not positive definite (i.e., $\left(T_{1}^{*}, T_{2}^{*}\right)$ is a saddle point in the 2-D threshold space) then at least one of the two thresholds is rejected. The appropriate one is eliminated by repeating the same test to the second partial derivatives $\partial^{2} J / \partial T_{1}^{2}$ and $\partial^{2} J / \partial T_{2}^{2}$ related to $T_{1}^{*}$ and $T_{2}^{*}$, respectively.

Due to its complexity, it is practically impossible to evaluate analytically the expression of the Hessian matrix from the criterion $J\left(T_{1}, T_{2}\right)$. We propose to compute it numerically by using simple approximations of the first- and second-order derivatives.

The whole procedure describing the selection of the number of thresholds is presented in the following:

Start

$$
\text { Step 1: Compute the Hessian matrix } H_{\left(T_{1}^{*}, T_{2}^{*}\right)}
$$

Step 2:

$$
\begin{aligned}
& \text { if } H_{\left(T_{1}^{*}, T_{2}^{*}\right)} \text { is positive definite, then } \\
& \qquad\left(T_{1}^{*}, T_{2}^{*}\right) \text { are both considered; } \\
& \text { elseif }\left(\partial^{2} J / \partial T_{1}^{2}\right) \leq 0 \text {, then } T_{1}^{*} \text { is eliminated; } \\
& \quad \text { elseif }\left(\partial^{2} J / \partial T_{2}^{2}\right) \leq 0 \text {, then } T_{2}^{*} \text { is eliminated; }
\end{aligned}
$$

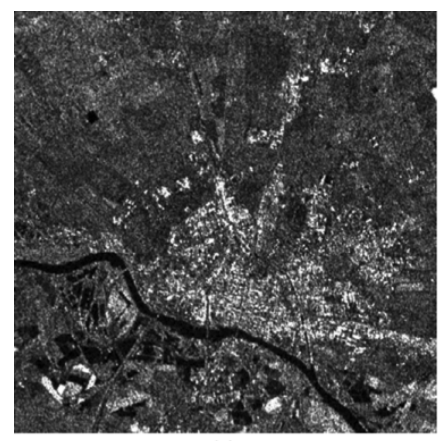

(a)

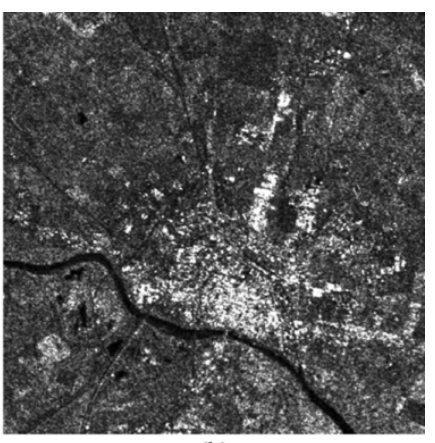

(b)

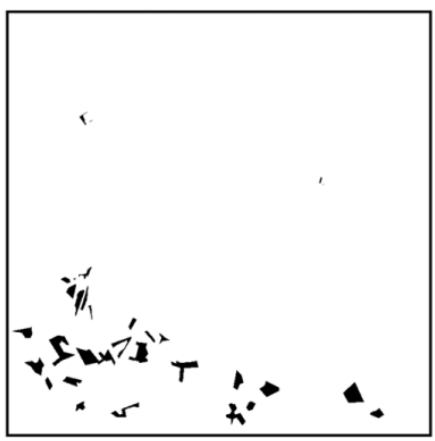

(c)

Fig. 3. Multitemporal real SAR images related to the city of Pavia. (a) Image acquired on October 20, 2000, immediately after the flooding. (b) Image acquired on October 28, 2000. (c) Ground-truth map.

If both thresholds are rejected, this implies that the histogram is viewed as unimodal by the double-thresholding $\mathrm{KI}$ algorithm (i.e., no changes occurred between the two acquisition dates of the two images).

\section{EXPERIMENTAL RESULTS}

In order to evaluate the effectiveness of the proposed method for the detection of the number and the values of the thresholds in the histogram, in the experiments we used two SAR images of size $730 \times 730$ acquired over the city of Pavia (Italy) on October 20 and October 28, 2000 [these images show an area affected by flooding (see Fig. 3)]. The equivalent number of looks (ENL) of the two images is 2.64 and 3.43, respectively, which means that this dataset is highly corrupted by speckle noise. To reduce this noise, we applied the enhanced Lee filter [3] with a window size $7 \times 7$ to the images. As in [1], also in this approach the optimal number of filtering iterations to be applied to the original images was determined automatically (in this case it was two for both real and simulated data). Since the aim of the letter is the automatic detection of the number of thresholds and their values, we refer the reader to [1] for greater detail on the automatic identification of the optimal number of filtering iterations.

\section{A. Results With a Single Change}

In the first experiment, we considered the original images (which describe a situation of a single change). The application of the modified double-thresholding KI algorithm to the histogram of the log-ratio image (see Fig. 4) yielded the two threshold values $(121,162)$. The corresponding Hessian matrix evaluated at this point is not positive definite (since its determinant is equal to zero), which means that $(121,162)$ is a saddle point with respect to $J\left(T_{1}, T_{2}\right)$. Consequently, we have to reject 


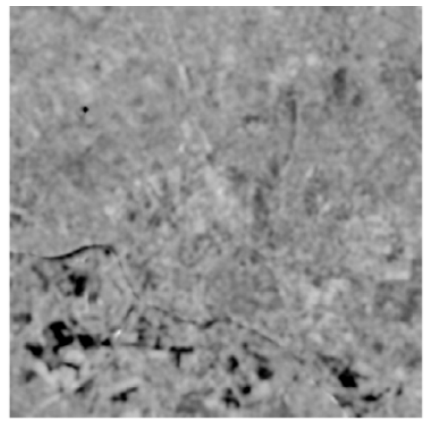

(a)

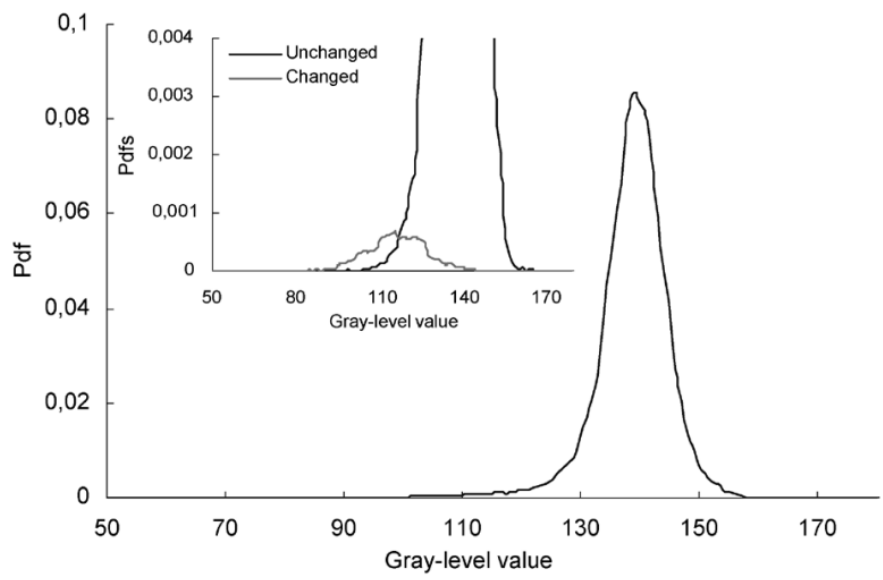

(b)

Fig. 4. (a) Log-ratio image obtained from filtered multitemporal real SAR images and (b) corresponding histogram for the experiment with one change. A zoom of the histogram shows changed and unchanged class distributions using the information in the available ground truth.

one of the two thresholds, which in this case is $T_{2}^{*}=162$ (because $\left.\partial^{2} J / \partial T_{2}^{2}\left(T_{1}^{*}, T_{2}^{*}\right)=0\right)$. In fact, the behavior of the criterion function $J\left(T_{1}, T_{2}\right)$ (see Fig. 5) clearly shows this situation since it is characterized by a minimum along $T_{1}$ and a saddle point along $T_{2}$, confirming the presence of a single change on one side of the unchanged class. Concerning the change-detection accuracy, the results, presented in terms of overall errors, false and missed alarms, obtained by the proposed automatic method are very close to those yielded by an optimal time-consuming manual trial and error procedure (MTEP) (see Table I). The latter consists of finding the threshold that minimizes the probability of error (on the basis of the available ground-truth) by trying all the $L$ possible threshold values. Moreover, a visual comparison between the change-detection maps obtained by both methods shows that they are very similar (see Fig. 6).

\section{B. Results With Double Change}

In the second experiment, we evaluated the effectiveness of the method in detecting two kinds of changes. To simulate such a situation, some pixels characterizing the flooded areas were exchanged between the two original SAR images. Thus, from the obtained simulated multitemporal SAR images, we generated the corresponding log-ratio image (see Fig. 7). The thresholds obtained by applying the modified double-thresholding KI algorithm to the log-ratio image were $(119,155)$. Both values are

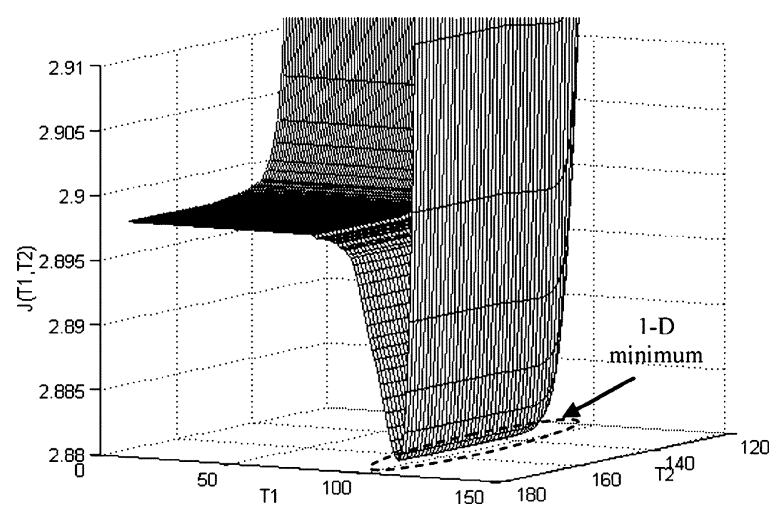

Fig. 5. Behavior of the criterion function $J\left(T_{1}, T_{2}\right)$ in the case of one change.

TABLE I

Results ObTained By the Proposed Method and the MTEP For the Real Multitemporal SAR Images (CASE of One Change)

\begin{tabular}{l|c|c|c|c}
\cline { 2 - 5 } & $T_{1}$ & $\begin{array}{c}\text { False } \\
\text { Alarms }\end{array}$ & $\begin{array}{c}\text { Missed } \\
\text { Alarms }\end{array}$ & $\begin{array}{c}\text { Overall } \\
\text { Error }\end{array}$ \\
\hline Proposed Method & 121 & 2170 & 3424 & 5594 \\
\hline MTEP & 119 & 1484 & 3901 & 5385 \\
\hline \hline
\end{tabular}

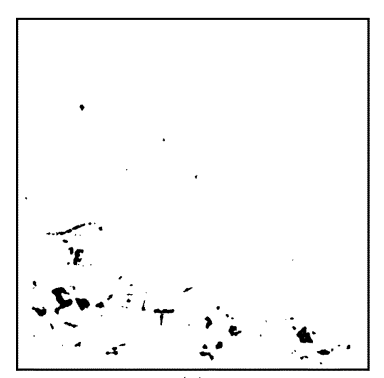

(a)

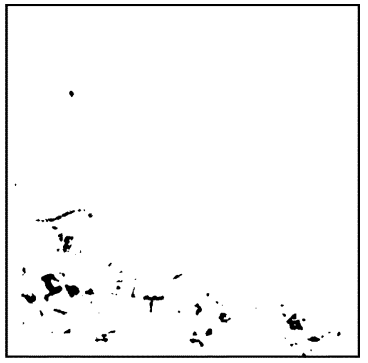

(b)
Fig. 6. Change-detection maps obtained (a) by the proposed method and (b) by the MTEP (case of one change).

considered since the Hessian matrix evaluated at this point is positive definite. In fact, the behavior of the criterion $J\left(T_{1}, T_{2}\right)$ (see Fig. 8) indicates the presence of such a minimum in the 2-D threshold space. In this case, the change-detection results provided by the proposed method were similar to those yielded by the MTEP (see Table II). This confirms the effectiveness of the proposed method in the detection of the number of thresholds and their values, and in the generation of an accurate change-detection map.

\section{Results With No Change}

In order to test the effectiveness of the proposed method in detecting a no-change situation, we selected a region $(250 \times 250$ pixels) from the original images, where no changes occurred between the two acquisition dates. In this case, the thresholds obtained by applying the modified double-thresholding KI algorithm were at the boundary of the cost function domain (i.e., 1 and 254). The Hessian matrix computed at this point is not symmetric positive definite, which means that both thresholds should be rejected. Moreover, the behavior of the criterion function $J\left(T_{1}, T_{2}\right)$ (see Fig. 9) confirmed the absence of a minimum, 


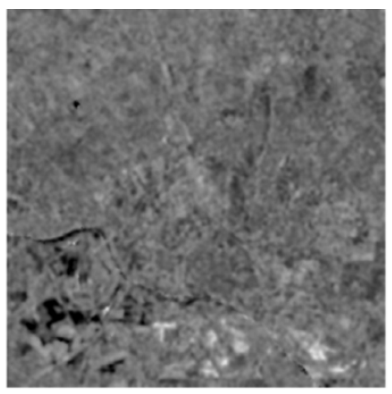

(a)

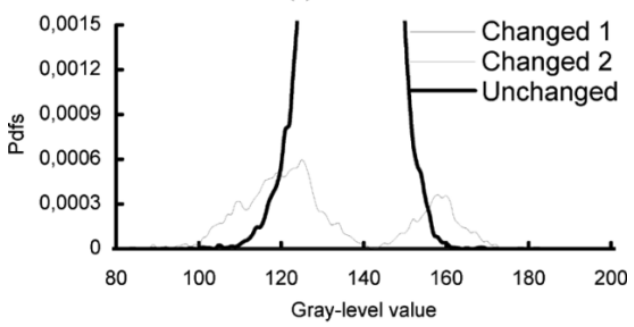

(b)

Fig. 7. (a) Log-ratio image obtained from the simulated multitemporal SAR images for the case of two changes and (b) corresponding changed and unchanged class distributions.

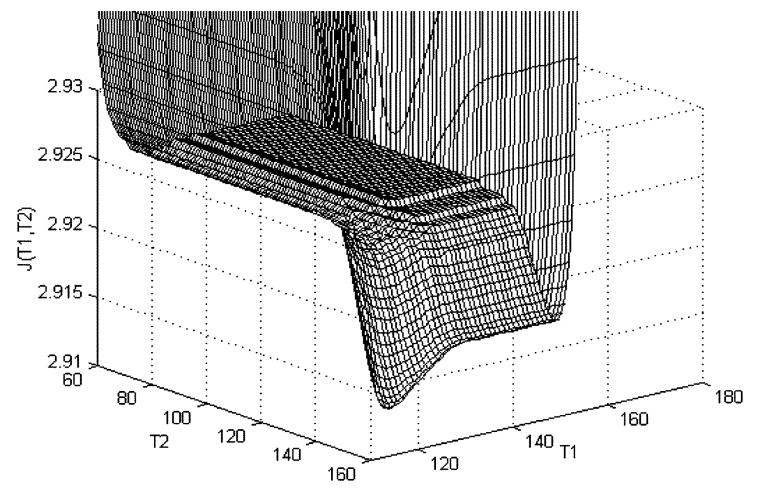

Fig. 8. Behavior of the criterion function $J\left(T_{1}, T_{2}\right)$ in the case of two changes.

TABLE II

RESUlts OBTAINED By THE PROPOSED METHOD AND THE MTEP FOR THE Simulated Multitemporal SAR IMAGes (CASE OF Two ChANGeS)

\begin{tabular}{l|c|c|c|c}
\cline { 2 - 5 } & $\left(T_{1}, T_{2}\right)$ & $\begin{array}{c}\text { False } \\
\text { Alarms }\end{array}$ & $\begin{array}{c}\text { Missed } \\
\text { Alarms }\end{array}$ & $\begin{array}{c}\text { Overall } \\
\text { Error }\end{array}$ \\
\hline Proposed Method & 119,155 & 1823 & 3591 & 5414 \\
\hline MTEP & 119,155 & 1823 & 3591 & 5414 \\
\hline \hline
\end{tabular}

which means that no reliable changes occurred between the two acquisition dates.

\section{CONCLUSION}

In this letter, an extension of the automatic approach to unsupervised change detection in multitemporal SAR images devel-

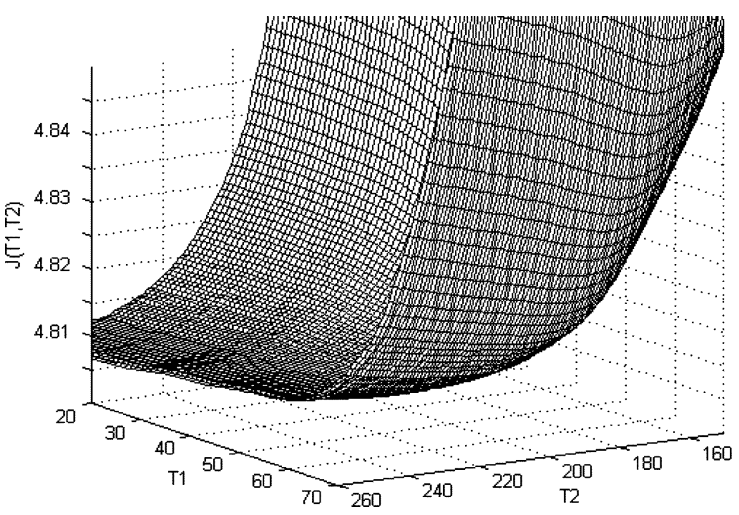

Fig. 9. Behavior of the criterion function $J\left(T_{1}, T_{2}\right)$ in the case of no change.

oped in [1] has been presented. Unlike traditional change-detection methods, with the proposed approach it is possible to determine automatically the number (zero, one, or two) and the values of the decision thresholds in the log-ratio image by analyzing a simple cost function. The latter is the minimum value of the criterion function obtained by applying the modified double-thresholding $\mathrm{KI}$ algorithm to the log-ratio image under the GG assumption for changed and unchanged classes. In this context, three decisions can be taken: 1) a situation of a double change is detected if the couple of thresholds estimated is a minimum with respect to the cost function in the 2-D threshold space; 2 ) a situation of a single change is obtained if the above couple is not a minimum in the 2-D threshold space (i.e., only one of the two thresholds is a minimum in its corresponding 1-D threshold space); 3) a situation of no change is observed if the thresholds are not minimum points both in the 2-D and the 1-D threshold spaces. The experimental results carried out on real and simulated data characterized by a single change, two changes, and no change at all confirmed the effectiveness of the proposed technique. It is noteworthy that the main limitation of the proposed method consists of its biased estimation of statistical parameters related to changed and unchanged classes, which may lead to the estimation of biased threshold values. It can be expected that this potential problem may be critical when changed and unchanged classes are strongly overlapped.

\section{ACKNOWLEDGMENT}

The authors thank P. Gamba (University of Pavia) for providing the multitemporal SAR images used in the experiments.

\section{REFERENCES}

[1] Y. Bazi, L. Bruzzone, and F. Melgani, "An unsupervised approach based on the generalized Gaussian model to automatic change detection in multitemporal SAR images," IEEE Trans. Geosci. Remote Sens., vol. 43, pp. 874-887, Apr. 2005.

[2] J. Kittler and J. Illingworth, "Minimum error thresholding," Pattern Recognit., vol. 19, pp. 41-47, 1986.

[3] A. Lopes, R. Touzi, and E. Nezry, "Adaptive speckle filters and scene heterogeneity," IEEE Trans. Geosci. Remote Sens., vol. 28, no. 6, pp. 992-1000, Nov. 1990. 